\title{
Respostas Fisiológicas e Produtivas de Vacas Holandesas em Lactação Submetidas a Diferentes Ambientes 1
}

\author{
Luciane Silva Martello², Holmer Savastano Júnior ${ }^{3}$, Saulo da Luz e Silva ${ }^{4}$, \\ Evaldo Antonio Lencioni Titto ${ }^{5}$
}

\begin{abstract}
RESUMO - Este trabalho foi realizado, no verão de 2002, com o objetivo de avaliar a influência de alguns recursos de climatização na produção de leite e na termorregulação dos animais. Foram utilizadas 10 primíparas e 17 multíparas em lactação, distribuídas em instalação controle (ICO), instalação com nebulizador associado a ventiladores (ICL) e instalação com tela de sombreamento (IT). A produção de leite e o consumo individual foram medidos diariamente para cada animal. A temperatura retal foi medida três vezes ao dia, em três animais de cada instalação; a temperatura de superfície da pele, três vezes ao dia em todos os animais; e a freqüência respiratória, duas vezes ao dia em todos os animais. Os dados climáticos de cada instalação foram registrados e posteriormente calculados os índices de temperatura e umidade (ITU) e de globo negro e umidade (ITGU). Foram selecionados e analisados 26 dias com entalpia elevada. O ITU entre 75 e 76, apesar de considerado estressante por diversas fontes da literatura, não foi associado à condição de estresse pelos animais. As multíparas da instalação climatizada apresentaram freqüência respiratória e temperatura de pele significativamente menor em relação às multíparas das demais instalações. As primíparas apresentaram freqüências respiratórias e temperaturas retais mais altas que as multíparas, nos horários mais quentes do dia. A maior produção de leite das multíparas foi observada no tratamento com tela.
\end{abstract}

Palavras-chave: estresse térmico, produção de leite, sistema de nebulização, temperatura retal, termorregulação

\section{Physiologic and Performance Responses of Holstein Cows in Milking under Different Environments}

\begin{abstract}
This work was carried out during the summer of 2002 to evaluate the influence of some cooling systems on the milk yield and animal thermoregulation. Ten heifers and seventeen milking cows were assigned to the control housing (ICO), mist \& fan housing (ICL) and shade cloth (80\%) (IT). The milk yield and the individual intake were daily measured for each animal. The rectal temperature was measured three times a day with three animals from each treatment. The skin surface temperature was collected three times a day for all the animals and the respiratory frequency two times a day for all the animals. The climatic data of each housing were registered to calculate the temperature humidity index (ITU) and the black globe humidity index (ITGU). Twenty six days of high enthalpy were selected and analyzed. The study showed that temperature humidity index from 75 to 76 was not associated with stress conditions for the animals, although many researches propose this situation as stressing. The milking cows in the mist \& fan treatment showed respiratory frequency and skin surface temperature significantly lower than the cows in the other treatments. The heifers presented respiratory frequency and rectal temperature higher than the cows in all the registration times. The higher milk yield of the cows was observed in the shade cloth treatment.
\end{abstract}

Key Words: heat stress, milk production, misting system, rectal temperature, thermoregulation

\section{Introdução}

A tendência das granjas leiteiras tem sido trabalhar com animais de alto potencial genético, concentrados em áreas cada vez menores. Esses animais, especializados em produção de leite, possuem metabolismo elevado, com produção de maior quantidade de calor endógeno (Titto, 1998). Essa afirmação justifica a crescente preocupação com o conforto animal, já que o Brasil é um país predominantemente de clima tropical, com altas temperaturas médias durante o ano, na maior parte do seu território, o que provoca o chamado estresse térmico.

Os ruminantes são animais classificados como homeotermos, ou seja, apresentam funções fisiológicas que se destinam a manter a temperatura corporal constante. Dentro de determinada faixa de temperatura ambiente, denominada zona de conforto ou de 
termoneutralidade, a manutenção da homeotermia ocorre com mínima mobilização dos mecanismos termorreguladores (Nääs, 1989). A mesma autora citou a faixa de 4 a $24^{\circ} \mathrm{C}$ como confortável para vacas em lactação, que pode se restringir aos limites de 7 e $21^{\circ} \mathrm{C}$, em razão da umidade relativa e da radiação solar.

A literatura mostra grande número de trabalhos sobre ambiência e conforto térmico em animais, porém são escassos os estudos que associam o ambiente com as respostas fisiológicas e produtivas. Os índices de conforto térmico, determinados por meio dos fatores climáticos, servem como indicativos para caracterizar o conforto e o bem-estar animal (Albright, 1993). As respostas fisiológicas ao estresse térmico mais utilizadas para o desenvolvimento dos índices são a temperatura corporal, a freqüência respiratória e o volume respiratório (Fehr et al., 1993).

$\mathrm{O}$ índice de temperatura e umidade (ITU), que relaciona temperatura e umidade relativa do ar, é o mais utilizado pelos pesquisadores para avaliação do estresse térmico. Johnson (1980) considerou que ITU a partir de 72 apresentava situação de estresse para vacas holandesas. $\mathrm{O}$ índice de temperatura de globo negro e umidade (ITGU), desenvolvido por Buffington et al. (1981) para avaliação do conforto térmico de vacas leiteiras expostas a ambientes com radiação solar direta e indireta, considera em seu cálculo a temperatura de globo negro e temperatura de ponto de orvalho.

Apesar de ser o meio natural de controle da temperatura do organismo, a termorregulação representa esforço extra e, conseqüentemente, alteração na produtividade. A manutenção da homeotermia é prioridade para os animais e impera sobre as funções produtivas como produção de leite, reprodução e produção de ovos.

O primeiro sinal visível de animais submetidos ao estresse térmico é o aumento da freqüência respiratória. Aumento ou diminuição da freqüência respiratória está na dependência da intensidade e da duração do estresse a que estão submetidos os animais. Esse mecanismo fisiológico promove a perda de calor por meio evaporativo. Segundo Hahn et al. (1997), a freqüência de 60 mov. $\mathrm{min}^{-1}$ indica animais com ausência de estresse térmico ou que este é mínimo.

Estudos confrontando vacas em lactação com exposição ao sol e animais totalmente sombreados relataram redução na freqüencia respiratória e aumento na produção de leite (Collier et al., 1981;
Roman-Ponce et al., 1977). Já Baccari Jr et al. (1982) observaram efeitos do sombreamento apenas sobre as variáveis fisiológicas (temperatura retal e freqüência respiratória), enquanto a produção de leite não variou. Segundo esses autores, este fato pode ter ocorrido em razão do baixo nível produtivo dos animais avaliados. Arcaro Jr. et al. (2001) também compararam vacas em lactação em três tipos de instalação: (i) sombra, (ii) sombra mais ventilação forçada e (iii) sombra com ventilação forçada associada à aspersão. Os autores relataram freqüência respiratória mais baixa dos animais na instalação com ventilação forçada e produção de leite mais alta para os animais submetidos à sombra com ventilação associada à aspersão.

A medida da temperatura retal é usada freqüentemente como índice de adaptabilidade fisiológica aos ambientes quentes, pois seu aumento mostra que os mecanismos de liberação de calor tornaram-se insuficientes (Mota, 1997). Segundo Kolb (1987), a temperatura retal média para bovinos com mais de um ano de idade é de $38,5 \pm 1,5^{\circ} \mathrm{C}$. Esta temperatura é mantida mediante regulação cuidadosa do equilíbrio entre a formação de calor e sua liberação pelo organismo.

Baccari Jr. et al. (1984) observaram que a temperatura retal média da tarde é, em geral, mais elevada que a da manhã. Baccari Jr. et al. (1979) constataram que a temperatura retal acompanhou a temperatura do ar até determinado horário e que, a partir de então, a temperatura retal continuou a subir, enquanto a temperatura do ar diminuía. Os autores concluíram que a temperatura retal guardou maior relação com a hora do dia do que com a temperatura do ar. Silva (2000), entretanto, relatou que, em razão das diferenças na atividade metabólica dos diversos tecidos, a temperatura não é homogênea no corpo todo e varia de acordo com a região anatômica.

Baccari Jr. et al. (1978) também estudaram a diferença entre a temperatura retal e a da pele em garrotes Chianina e observaram que a temperatura média da pele foi mais baixa que a retal. Da mesma forma, Cappa et al. (1989) e Aguiar (1999) observaram que a temperatura da pele foi mais baixa que a retal em vacas em lactação.

Vacas leiteiras, sob condições ambientais termicamente desconfortantes, tendem a reduzir consideravelmente o consumo de matéria seca (MS), na tentativa de diminuir a taxa metabólica e a conseqüente produção de calor metabólico (Collier \& Beede, 
1985). Fortes evidências indicam que a redução no consumo voluntário de alimentos tem sido a principal razão dos decréscimos na produção de leite em vacas submetidas ao estresse pelo calor (Chen et al., 1993; McGuire et al., 1989; McDowell et al., 1976 e Maust et al., 1972). Trabalho de Damasceno \& Targa (1998), no entanto, resultou em ausência de efeitos da modificação ambiental no consumo de MS, embora a produção de leite tenha sido sensivelmente afetada. Furquay (1997), avaliando o efeito da ventilação em vacas lactantes, em clima quente, observou maiores valores de temperatura retal para as vacas do grupo sem ventiladores, sem diferença para a produção de leite.

Maust et al. (1972) e Johnson (1982) demonstraram que o estresse térmico afetou o consumo de matéria seca no mesmo dia. De acordo com os autores, o estresse pelo calor aumenta a temperatura corporal, que deprime a ingestão de alimentos no mesmo dia e reduz a produção de leite poucos dias depois. Schneider et al. (1984) observaram que vacas com acesso à sombra ingeriram mais alimento e produziram mais leite que suas pares sem sombra.

Outros trabalhos, como os de Roman-Ponce et al. (1977), Collier et al. (1981) e Damasceno \& Targa (1998), também demonstraram a superioridade da produção de leite de vacas com acesso à sombra, comparada à de vacas expostas a radiação solar direta ou a sombra restrita. De acordo com Cardoso et al. (1983), que trabalharam com vacas holandesas, o uso de sombra parcial foi adequado para abrigar estes animais em comparação ao uso de sombra total.

Nääs \& Arcaro Jr. (2001) avaliaram o efeito da ventilação associada a aspersão em instalações para vacas lactantes. Os autores relataram menores valores de temperatura retal e de freqüência respiratória e maiores valores de produção de leite para o grupo de animais que estava alojado nestas instalações, em comparação ao grupo sem acesso a ventiladores e aspersores.

O objetivo do presente trabalho foi avaliar a possível influência do conforto proporcionado pelas diferentes instalações na produção de leite e na termorregulação de novilhas e vacas.

\section{Material e Métodos}

O trabalho foi realizado no Setor de Bovinocultura de Leite da Prefeitura do Campus Administrativo da Universidade de São Paulo em Pirassununga (PCAPS), no período de 18 de fevereiro a 21 de março de 2002. O período de adaptação dos animais às instalações e ao manejo antecedeu em 10 dias o início do experimento.

Foram utilizados animais da raça Holandesa preta e branca: 17 multíparas com média de produção de $5500 \mathrm{~kg}$ leite/lactação e 10 primíparas entre o segundo e o sétimo mês do estádio de lactação. Para cada tratamento (instalação), foram distribuídos, por sorteio, animais das duas categorias. Na Figura 1, apresentam-se, de forma esquemática, o posicionamento e as dimensões das instalações do experimento. A instalação controle (ICO) contou com o sombreamento de $37,2 \mathrm{~m}^{2}$ oferecido pela cobertura do cocho com telha de cimento-amianto. $\mathrm{Na}$ instalação climatizada (ICL), foram instalados dois ventiladores e um nebulizador de baixa pressão (mist) sobre os cochos na área coberta. Os ventiladores foram programados para ligar a partir da temperatura do ar de $24^{\circ} \mathrm{C}$ e os nebulizadores eram acionados a partir da temperatura de $26^{\circ} \mathrm{C}$. No piquete da instalação com tela (IT), foi instalada uma cobertura de $60 \mathrm{~m}^{2}$, com tela preta de polietileno com malha para $80 \%$ de sombra, colocada em camada única sobre estrutura de madeira, sem fechamento lateral e com pé-direito de $3,5 \mathrm{~m}$. As três instalações apresentavam área com piquete adjacente descoberto de $362 \mathrm{~m}^{2}$ e características iguais no que se refere à estrutura construtiva da área de cocho. Os cochos eram equipados com sistema de portão eletrônico, do tipo Calan Gate (Calan Systems Inc.), que permitiu o controle do consumo alimentar individual. Foi fornecida a mesma ração, ad libitum, em todos os tratamentos. A relação volumoso: concentrado foi de 58:42 e o concentrado, balanceado com $18 \%$ de proteína bruta e $70 \%$ de nutrientes digestíveis totais (NDT).

Para cada tratamento foram calculados os índices ITU e ITGU, descritos abaixo:

$\mathrm{ITU}=\mathrm{Tbs}+0,36 \mathrm{To}+41,2$.

$\mathrm{ITGU}=\mathrm{Tg}+0,36 \mathrm{To}+41,5$

em que $\mathrm{Tbs}=$ temperatura de bulbo seco $\left({ }^{\circ} \mathrm{C}\right)$; To = temperatura do ponto de orvalho $\left({ }^{\circ} \mathrm{C}\right)$; $\mathrm{Tg}=$ temperatura do termômetro de globo negro $\left({ }^{\circ} \mathrm{C}\right)$.

Para análise dos dados, selecionaram-se os dias em que a entalpia, variável física que indica a quantidade de calor contida em uma mistura de ar seco, das $13 \mathrm{~h}$, horário mais quente do dia, do tratamento controle, esteve acima da considerada crítica. Consi- 




Figura 1 - Esquema e dimensões das instalações controle (ICO), climatizada (ICL) e tela (IT). Figure 1 - Layout and dimensions of control (ICO), cooling (ICL) and shade cloth (IT) housing. Medidas em metros. Sem escala.

Dimensions unit: metres. No escale.

derou-se crítica a entalpia a partir de $63,51 \mathrm{~kJ} / \mathrm{kg}$ de ar seco, obtida a partir da temperatura de $24^{\circ} \mathrm{C}$ e da umidade relativa do ar de $76 \%$ (Johnson \& Vanjonack, 1976, citados por Baccari Jr., 1998). Foram observados 26 dias nessa condição e toda análise refere-se a esses dias. Para o cálculo utilizou-se o programa computacional PsyCalc $98^{\circledR}$ (Monterrey Institute of Technology), que considera a temperatura de bulbo seco $\left({ }^{\circ} \mathrm{C}\right)$, a umidade relativa do ar $(\%)$ e a pressão barométrica local, igual a $603,14 \mathrm{~mm} \mathrm{Hg}$.

Foram registradas as temperaturas retal (TR) e de superfície da pele (TP), bem como a freqüência respiratória (FR). A TR foi coletada diariamente, em três animais de cada instalação, às 6,13 e 17 h. Para medida da TP, foi utilizado termômetro de infravermelho a uma distância máxima de $10 \mathrm{~m}$, em todos os animais às $6,13 \mathrm{e} 17 \mathrm{~h}$. A FR foi tomada pela contagem dos movimentos respiratórios (flanco) por meio de coletas diárias às $13 \mathrm{e} 17 \mathrm{~h}$ em todos os animais. Registraram-se também a produção de leite (PL) e o consumo de matéria seca (MS) individuais e diários.

O delineamento experimental adotado foi o inteiramente casualizado. Os dados de termorregulação e de produção de leite foram analisados em arranjo fatorial 3 x 2 (tratamento x categoria animal) pelo procedimento Mixed do programa SAS (1990).

\section{Resultados e Discussão}

Os valores médios, dos 26 dias do experimento, de entalpia e de ITU (Tabela 1), em todas as instala- 
ções, estiveram acima do considerado crítico por alguns autores, como Johnson (1980), que considerou que ITU a partir de 72 apresentava situação de estresse para vacas holandesas.

Em relação aos dados fisiológicos (Tabela 2), as médias de TR, às 6 e $17 \mathrm{~h}$, não foram diferentes entre as categorias. Às $13 \mathrm{~h}$, as primíparas apresentaram TR maior $(\mathrm{p}<0,01)$, se comparada à das multíparas.

Com exceção das $6 \mathrm{~h}$, os valores absolutos de TR das primíparas foram maiores que os das multíparas, o que poderia indicar que aquela categoria foi menos tolerante às condições ambientais do experimento. No entanto, provavelmente as amplitudes térmicas $\left(10,1,6,1\right.$ e $11,5^{\circ} \mathrm{C}$, nas instalações ICO, ICL e IT, respectivamente) facilitaram a perda de calor durante a noite, o que permitiu às primíparas manutenção da TR dentro da faixa de conforto considerada normal (Silva, 2000 e Baccari Jr. et al. 1984).

Às 13 e $17 \mathrm{~h}$, a FR das primíparas foi maior $(\mathrm{p}<0,01)$ que a das multíparas. Nos dois horários, as primíparas apresentaram FR acima da considerada normal (18 a 60 mov. $\left.\mathrm{min}^{-1}\right)$ por alguns autores

Tabela 1 - Médias diárias dos 26 dias críticos das variáveis climáticas e dos índices de conforto térmico nas instalações controle (ICO), climatizada (ICL) e tela (IT)

Table 1 - Climatic variables and thermal comfort indexes averages of 26 critical days in control (ICO), cooling (ICL) and shade cloth (IT) housing

\begin{tabular}{lccc}
\hline Variáveis & \multicolumn{3}{c}{$\begin{array}{c}\text { Tratamentos } \\
\text { Treatments }\end{array}$} \\
\cline { 2 - 4 } & ICO & ICL & IT \\
\hline $\begin{array}{l}\text { Temperatura de bulbo seco, }{ }^{\circ} \mathrm{C} \\
\text { Dry bulb temperature, }{ }^{\circ} \mathrm{C}\end{array}$ & $27,3^{\mathrm{a}}$ & $26,3^{\mathrm{b}}$ & $27,5^{\mathrm{a}}$ \\
$\begin{array}{l}\text { Umidade relativa, } \% \\
\text { Relative humidity, \% }\end{array}$ & $68,2^{\mathrm{a}}$ & $78,1^{\mathrm{b}}$ & $61,0^{\mathrm{c}}$ \\
$\begin{array}{l}\text { Temperatura de globo negro, }{ }^{\circ} \mathrm{C} \\
\text { Black globe temperature, }{ }^{\circ} \mathrm{C}\end{array}$ & $28,3^{\mathrm{a}}$ & $25,8^{\mathrm{b}}$ & $29,3^{\mathrm{a}}$ \\
Entalpia, kJ/kg de ar seco & & & \\
Enthalpy, $k J / k g$ of dry air & $70,7^{\mathrm{a}}$ & $69,2^{\mathrm{ab}}$ & $66,7^{\mathrm{b}}$ \\
ITU $^{1}$ & & & \\
ITGU $^{2}$ & $76,0^{\mathrm{a}}$ & $75,0^{\mathrm{a}}$ & $75,5^{\mathrm{a}}$ \\
\hline
\end{tabular}

$a, b, c$ Médias seguidas de letras distintas na mesma linha diferem $(p<0,01)$ pelo teste de Bonferroni.

$a, b, c$ Averages followed by distinct letters in the same row differ $(p<.01)$ by Bonferron test.

1 Índice de temperatura e umidade.

1 Temperature and humidity index.

2 Índice de temperatura de globo e umidade.

2 Black globe humidity index.

Tabela 2 - Médias das variáveis fisiológicas de cada categoria em diferentes horários, de todos os tratamentos.

Table 2 - Average of physiological variables to each category at different day time of all treatments.

\begin{tabular}{|c|c|c|c|c|}
\hline \multirow{2}{*}{$\begin{array}{l}\text { Variáveis } \\
\text { Variables }\end{array}$} & \multirow{2}{*}{$\begin{array}{l}\text { Categorias } \\
\text { Categories }\end{array}$} & \multicolumn{2}{|c|}{$\begin{array}{c}\text { Horários } \\
\text { Day times }\end{array}$} & \\
\hline & & $6: 00$ & $13: 00$ & $17: 00$ \\
\hline Temperatura retal ${ }^{\circ} \mathrm{C}$ & Primípara & $38,3^{\mathrm{a}}$ & $39,1^{\mathrm{a}}$ & $39,3^{\mathrm{a}}$ \\
\hline Rectal temperature, ${ }^{\circ} \mathrm{C}$ & $\begin{array}{c}\text { Heifer } \\
\text { Multípara } \\
\text { Cow }\end{array}$ & $38,3^{\mathrm{a}}$ & $38,8^{\mathrm{b}}$ & $39,0^{\mathrm{a}}$ \\
\hline $\begin{array}{l}\text { Freqüência respiratória, mov. } \min ^{-1} \\
\text { Respiratory rate, }{\text { mov. } \mathrm{min}^{-1}}^{-1}\end{array}$ & $\begin{array}{l}\text { Primípara } \\
\text { Heifer } \\
\text { Multípara } \\
\text { Cow }\end{array}$ & - & $68,0^{\mathrm{a}}$ & $62,4^{\mathrm{a}}$ \\
\hline
\end{tabular}

aa,b Médias seguidas de letras distintas na mesma linha diferem $(p<0,01)$ pelo teste de Bonferroni.

$\mathrm{a}, \mathrm{b}$ Averages followed by distinct letters in the same row differ $(p<.01)$ by Bonferron test. 
(Hahn et al., 1997; Andersson \& Jónasson, 1996), enquanto as multíparas não apresentaram indicativos de estresse em nenhum dos horários analisados.

Assim como na análise da TR, observou-se a menor tolerância das primíparas ao ambiente climático em comparação às multíparas, pois a FR das primeiras indicou condição de estresse mínimo.

$\mathrm{Na}$ análise dos dados das variáveis fisiológicas em cada instalação e horário (Tabela 3 ), observou-se que, somente às $6 \mathrm{~h}$, a TR dos animais apresentou alguma diferença entre os tratamentos. A TR média no tratamento ICL foi maior $(\mathrm{p}<0,01)$ que nos tratamentos ICO e IT. Porém, o maior valor para o ICL não implicou a ocorrência de estresse, uma vez que biologicamente estes valores são considerados normais para vacas em lactação.

Os valores numéricos de TR da tarde (13 e $17 \mathrm{~h})$ foram superiores aos da manhã em todos os tratamentos. Baccari Jr. et al. (1979) relataram que, em condições de termoneutralidade, a TR de bovinos guardou relação mais alta com a hora do dia, do que com a temperatura de bulbo seco (Tbs) no transcorrer do dia. Isso também pôde ser observado neste trabalho, uma vez que a Tbs e a TR, para todos os tratamentos, aumentaram até as $13 \mathrm{~h}$ e, a partir de então, a Tbs declinou, enquanto a TR continuou a subir.

As temperaturas retais, de todos os tratamentos e em todos os horários, estiveram dentro da faixa considerada normal $\left(37,5\right.$ a $\left.39,3^{\circ} \mathrm{C}\right)$ por Silva (2000), o que indica que os sistemas de climatização e de sombreamento adotados neste experimento foram suficientes, sob esse critério, para manter a condição de conforto térmico.
Em análise mais ampla, os resultados de TR, dentro da faixa normal de conforto, indicaram que os valores médios de ITU entre 75 e 76 não ocasionaram condição de estresse aos animais. Estes resultados estão de acordo com Lemerle \& Goddard (1986), que relataram aumento da TR para ITU acima de 80 .

Provavelmente, o microclima proporcionado pelo efeito dos ventiladores e dos nebulizadores, no ICL, resultou a menor $(p<0,01)$ FR dos animais ali abrigados, se comparados àqueles do ICO e IT. Estes dois últimos tratamentos não apresentaram diferenças significativas entre si nos dois horários. Tais resultados estão de acordo com os de Nääs \& Arcaro Jr. (2001), que observaram menor FR de vacas lactantes, em ambiente com ventilação e aspersão d'água, comparadas a vacas em ambiente sem climatização, com valores de 42,8 e 47,6 mov. $\mathrm{min}^{-1}$ respectivamente.

A análise dos dados de TP demonstrou a ocorrência de interação tripla entre hora, tratamento e categoria animal $(\mathrm{p}<0,01)$. Por isso, as categorias foram avaliadas separadamente (Tabela 4 ).

Às $13 \mathrm{~h}$, a TP média das primíparas para o tratamento IT foi menor $(\mathrm{p}<0,01)$ que a TP no tratamento ICL. Para as multíparas, no mesmo horário, a TP no tratamento ICL foi menor $(p<0,01)$ do que nos tratamentos ICO e IT. Estes dois últimos tratamentos não diferiram estatisticamente entre si.

No horário mais quente, às $13 \mathrm{~h}, \mathrm{o}$ comportamento das multíparas, em relação à TP, foi diferente daquele das primíparas. Nesse horário, para o tratamento ICL, em relação aos demais tratamentos, as primíparas apresentaram a maior média de $\mathrm{TP}$, em

Tabela 3 - Médias das variáveis fisiológicas das multíparas e primíparas nos tratamentos controle (ICO), climatizada (ICL) e tela (IT) nos diferentes horários

Table 3 - Average of physiological variables of cows and calves in control (ICO), cooling (ICL) and shade cloth (IT) at different day times

\begin{tabular}{|c|c|c|c|c|}
\hline \multirow{2}{*}{$\begin{array}{l}\text { Variáveis } \\
\text { Variables }\end{array}$} & \multirow{2}{*}{$\begin{array}{l}\text { Tratamentos } \\
\text { Treatments }\end{array}$} & \multicolumn{3}{|c|}{$\begin{array}{l}\text { Horários } \\
\text { Day times }\end{array}$} \\
\hline & & $6: 00$ & $13: 00$ & $17: 00$ \\
\hline \multirow{3}{*}{$\begin{array}{l}\text { Temperatura retal, }{ }^{\circ} \mathrm{C} \\
\text { Rectal temperature, }{ }^{\circ} \mathrm{C}\end{array}$} & ICO & $38,2^{b}$ & $38,9^{\mathrm{a}}$ & $39,2^{\mathrm{a}}$ \\
\hline & ICL & $38,5^{\mathrm{a}}$ & $39,0^{\mathrm{a}}$ & $39,2^{\mathrm{a}}$ \\
\hline & IT & $38,2^{b}$ & $39,0^{\mathrm{a}}$ & $39,2^{\mathrm{a}}$ \\
\hline \multirow{3}{*}{$\begin{array}{l}\text { Freqüência respiratória, mov. } \mathrm{min}^{-1} \\
\text { Respiratory rate, }{\text { mov. } \mathrm{min}^{-1}}^{-1}\end{array}$} & ICO & - & $63,4^{\mathrm{a}}$ & $61,6^{\mathrm{a}}$ \\
\hline & ICL & - & $59,0^{\mathrm{b}}$ & $52,4^{\mathrm{b}}$ \\
\hline & IT & - & $66,7^{\mathrm{a}}$ & $63,0^{\mathrm{a}}$ \\
\hline
\end{tabular}

a,b Médias seguidas de letras distintas na mesma linha diferem $(p<0,01)$ pelo teste de Bonferroni.

$\mathrm{a}, \mathrm{b}$ Averages followed by distinct letters in the same row differ $(p<.01)$ by Bonferron test. 
valores absolutos, enquanto as multíparas apresentaram a menor média. Isso pode indicar que, nos horários de temperatura mais elevada do dia, as primíparas não se beneficiaram do sistema de aspersão. Uma explicação possível é que tenha ocorrido certa dominância das multíparas com relação à disputa por espaço no local climatizado do tratamento ICL, uma vez que a sombra disponibilizada foi somente a da cobertura do cocho $\left(2,5 \mathrm{~m}^{2} /\right.$ animal $)$. Conforme discutido anteriormente, os valores de TR e FR para as primíparas, às $13 \mathrm{~h}$, também foram superiores aos das multíparas (Tabela 2), o que pode reforçar esta explicação. Outro fato importante foi que a TP das primíparas, neste mesmo horário, foi menor no tratamento IT, cuja disponibilidade de sombra por animal era maior ( $9 \mathrm{~m}^{2}$ /animal).

A TP, associada a outros parâmetros fisiológicos, está sendo utilizada por alguns pesquisadores como indicativo de estresse térmico em animais (Aguiar, 1999; Cappa et al., 1989; Baccari Jr. et al., 1978). Segundo Baccari Jr. (2001), existe um gradiente térmico no organismo, de modo que a temperatura é mais elevada no seu interior e diminui até a periferia (pele e pêlos).

De acordo com os dados médios de TP e TR das primíparas, mostrados na Tabela 5, observou-se que os gradientes térmicos retais-cutâneos foram de 4,3, 5,4 e $4,7^{\circ} \mathrm{C}$ para ICO, ICL e IT, respectivamente. Para as multíparas, os gradientes foram 4,6, 5,8 e $4,3^{\circ} \mathrm{C}$ para ICO, ICL e IT nesta mesma ordem. Estes dados estão próximos aos relatados por Baccari Jr. et al. (1978) e Aguiar (1999), que encontraram gradientes térmicos retais-cutâneos de 4 e $6,3^{\circ} \mathrm{C}$, sendo a temperatura de pele mais baixa que a retal.
Os dados de FR indicaram condição de estresse mínimo para as primíparas. Esses resultados sugerem a necessidade de estudos adicionais das diferenças de respostas fisiológicas entre primíparas e multíparas submetidas ao mesmo ambiente físico. Tais estudos poderão auxiliar, futuramente, nas técnicas de manejo ambiental mais adequadas para as diferentes categorias presentes nos rebanhos.

Em análise mais ampla, os resultados de TR e FR indicaram que médias diárias de ITU até 76,0 e de ITGU até 77,8 não causaram alteração na condição normal da termorregulação dos animais. Tal fato sugere investigações adicionais dos limites críticos destes índices para vacas em lactação criadas em regiões tropicais.

As observações dos efeitos climáticos e de termorregulação sobre os resultados produtivos consideraram as médias diárias das variáveis climáticas e de termorregulação (Tabelas 1 e 5).

A ingestão de matéria seca (IMSPM) foi avaliada com base no peso metabólico dos animais (Tabela 6). $\mathrm{Na}$ categoria das primíparas, o tratamento ICO apresentou IMSPM maior $(p<0,01)$ que do tratamento IT. Não houve diferença entre os tratamentos ICL e IT e nem entre ICL e ICO.

As médias de IMSPM das multíparas, nos tratamentos IT e ICO, não foram significativamente diferentes entre si. $\mathrm{O}$ tratamento ICL apresentou menor consumo $(p<0,01)$ em relação aos demais tratamentos.

De acordo com Collier \& Beede (1985), sob condições de estresse térmico, os animais reduzem o consumo de alimento, na tentativa de diminuir a taxa metabólica e a produção de calor endógeno.

Conforme já exposto, a análise da FR das

Tabela 4 - Médias de temperatura da pele das categorias nos tratamentos controle (ICO), climatizado (ICL) e tela (IT) nos diferentes horários

Table 4 - Surface temperature averages to each category in control (ICO), cooling (ICL) and shade cloth (IT) at different day times

\begin{tabular}{|c|c|c|c|c|c|}
\hline \multirow{2}{*}{$\begin{array}{l}\text { Variável } \\
\text { Variables }\end{array}$} & \multirow{2}{*}{$\begin{array}{c}\text { Categorias } \\
\text { Categories }\end{array}$} & \multirow{2}{*}{$\begin{array}{l}\text { Horários } \\
\text { Day times }\end{array}$} & \multicolumn{3}{|c|}{$\begin{array}{c}\text { Tratamentos } \\
\text { Treatments }\end{array}$} \\
\hline & & & ICO & ICL & IT \\
\hline \multirow{2}{*}{$\begin{array}{l}\text { Temperatura de pele, }{ }^{\circ} \mathrm{C} \\
\text { Surface temperature, }{ }^{\circ} \mathrm{C}\end{array}$} & $\begin{array}{l}\text { Primípara } \\
\text { Heifer }\end{array}$ & $\begin{array}{c}6: 00 \\
13: 00 \\
17: 00\end{array}$ & $\begin{array}{c}32,1^{\mathrm{a}} \\
36,7^{\mathrm{ac}} \\
35,7^{\mathrm{a}}\end{array}$ & $\begin{array}{l}31,4^{\mathrm{a}} \\
37,7^{\mathrm{a}} \\
34,0^{\mathrm{b}}\end{array}$ & $\begin{array}{c}32,1^{\mathrm{a}} \\
36,0^{\mathrm{bc}} \\
35,3^{\mathrm{a}}\end{array}$ \\
\hline & $\begin{array}{l}\text { Multípara } \\
\text { Cow }\end{array}$ & $\begin{array}{c}6: 00 \\
13: 00 \\
17: 00\end{array}$ & $\begin{array}{l}31,6^{\mathrm{a}} \\
36,3^{\mathrm{a}} \\
35,8^{\mathrm{a}}\end{array}$ & $\begin{array}{l}31,6^{\mathrm{a}} \\
34,7^{\mathrm{b}} \\
33,8^{\mathrm{b}}\end{array}$ & $\begin{array}{l}32,3^{\mathrm{a}} \\
36,2^{\mathrm{a}} \\
35,6^{\mathrm{a}}\end{array}$ \\
\hline
\end{tabular}

a, b, c Médias seguidas de letras distintas na mesma linha diferem $(p<0,01)$ pelo teste de Bonferroni.

$a, b, c$ Averages followed by distinct letters in the same row differ $(p<.01)$ by Bonferron test. 
primíparas (acima de 60 mov.min ${ }^{-1}$ ) mostrou condição de estresse em todos os tratamentos. Porém, esse fato parece não ter interferido no consumo, uma vez que o maior consumo e a maior $\mathrm{FR}$ foram observados no tratamento ICO, enquanto o ICL apresentou o menor consumo e a menor FR. Isso pode indicar que os níveis moderados de estresse térmico, como os demonstrados neste trabalho, não afetam o consumo de matéria seca.

De forma semelhante, Damasceno \& Targa (1998) não encontraram diferenças no consumo de animais em instalações com sombreamento total ou parcial, no período do verão, embora os dados de TR e FR tenham indicado condição de estresse térmico maior do que a do presente trabalho.

Por outro lado, estudos como o de Maust et al. (1972) e de Johnson (1982) demonstraram que o estresse térmico afetou o consumo de alimentos. Schneider et al. (1984) também relataram reduções de $23 \%$ no consumo, em razão do estresse térmico. Porém, nesses trabalhos, havia evidências da condição de estresse térmico.

Os dados médios de TR e FR das multíparas indicaram ausência de estresse térmico em todas as instalações e sinalizaram que, possivelmente, as diferenças no consumo foram influenciadas mais intensamente por outras variáveis que não as climáticas.

Pela análise das variáveis climáticas, observou-se que o ITU médio do ICL foi 1,3 e $0,7 \%$ menor que o do ICO e do IT, respectivamente (Tabela 1). Porém, o consumo do ICL não foi maior em nenhuma das categorias. Esse fato indica que, possivelmente, essas reduções foram muito pequenas, de forma a não interferirem nos resultados de consumo.

Outra explicação para a ausência dos efeitos climáticos sobre o consumo poderia estar no fato de os valores de ITU, em todas as instalações, permanecerem acima do crítico (72) para vacas em lactação, indicando que o efeito do estresse no consumo já estaria presente em todos os animais. Entretanto, esta explicação seria menos provável, uma vez que os resultados da termorregulação mostraram que não houve sinal de estresse térmico nos animais ou que este foi mínimo.

Os dados de produção de leite para a categoria das primíparas mostraram que os animais do tratamento ICO apresentaram maior produção $(p<0,01)$ que os dos tratamentos ICL e IT. Entre os tratamentos ICL e IT, não foram observadas diferenças estatisticamente significativas. Provavelmente, o sombreamento do tratamento ICO foi suficiente para que não ocorressem reduções no consumo e na produção de leite.

A PL das multíparas no tratamento IT apresentou produção maior $(\mathrm{p}<0,01)$ que as dos tratamentos ICL e ICO. Já as médias de PL de ICO e ICL não foram significativamente diferentes entre si.

Vários pesquisadores constataram que a principal razão para a redução da produção de leite em

Tabela 5 - Médias das variáveis fisiológicas de todos os horários para os 26 dias analisados nos tratamentos controle (ICO), climatizado (ICL) e tela (IT)

Table 5 - Averages of physiological variables of all day times for 26 analyzed days in control (ICO), cooling (ICL) and shade cloth (IT) treatments

\begin{tabular}{|c|c|c|c|c|}
\hline \multirow{2}{*}{$\begin{array}{l}\text { Variáveis } \\
\text { Variables }\end{array}$} & \multirow{2}{*}{$\begin{array}{c}\text { Categorias } \\
\text { Categories }\end{array}$} & \multicolumn{3}{|c|}{$\begin{array}{c}\text { Tratamentos } \\
\text { Treatments }\end{array}$} \\
\hline & & $\mathrm{ICO}$ & ICL & IT \\
\hline Temperatura retal, ${ }^{\circ} \mathrm{C}$ & $\begin{array}{l}\text { Primípara } \\
\text { Heifer }\end{array}$ & $38,5^{\mathrm{a}}$ & $38,8^{\mathrm{b}}$ & $38,6^{\mathrm{ab}}$ \\
\hline Rectal temperature, ${ }^{\circ} \mathrm{C}$ & $\begin{array}{c}\text { Multípara } \\
\text { Cow }\end{array}$ & $38,4^{\mathrm{a}}$ & $38,6^{\mathrm{a}}$ & $38,4^{\mathrm{a}}$ \\
\hline Temperatura de pele, ${ }^{\circ} \mathrm{C}$ & $\begin{array}{l}\text { Primípara } \\
\text { Heifer }\end{array}$ & $34,2^{a}$ & $33,4^{\mathrm{b}}$ & $33,9^{\mathrm{ab}}$ \\
\hline Surface temperature, ${ }^{\circ} \mathrm{C}$ & $\begin{array}{c}\text { Multípara } \\
\text { Cow }\end{array}$ & $33,8^{\mathrm{a}}$ & $32,8^{\mathrm{b}}$ & $34,1^{\mathrm{a}}$ \\
\hline Freqüência respiratória, mov.min ${ }^{-1}$ & $\begin{array}{l}\text { Primípara } \\
\text { Heifer }\end{array}$ & $70,2^{\mathrm{a}}$ & $61,6^{b}$ & $65,5^{\mathrm{ab}}$ \\
\hline Respiratory rate, mov. $\mathrm{min}^{-1}$ & $\begin{array}{l}\text { Multípara } \\
\text { Cow }\end{array}$ & $59,9^{\mathrm{a}}$ & $51,2^{b}$ & $60,3^{\mathrm{a}}$ \\
\hline
\end{tabular}

a,b Médias seguidas de letras distintas na mesma linha diferem $(p<0,01)$ pelo teste de Bonferroni.

a, b Averages followed by distinct letters in the same row differ $(p<.01)$ by Bonferron test. 
Tabela 6 - Médias de ingestão de matéria seca e de produção de leite de cada categoria nos tratamentos controle (ICO), climatizado (ICL) e tela (IT)

Table 6 - Averages of dry matter feed intake and of milk yield to each category in control (ICO), cooling (ICL) and shade cloth (IT) treatments

\begin{tabular}{|c|c|c|c|c|}
\hline \multirow{2}{*}{$\begin{array}{l}\text { Variáveis } \\
\text { Variables }\end{array}$} & \multirow{2}{*}{$\begin{array}{l}\text { Categorias } \\
\text { Categories }\end{array}$} & \multicolumn{3}{|c|}{$\begin{array}{c}\text { Tratamentos } \\
\text { Treatments }\end{array}$} \\
\hline & & ICO & ICL & IT \\
\hline \multirow{2}{*}{$\begin{array}{l}\text { Ingestão de material seca, } \mathrm{kg} \text { MS } / \mathrm{kg} \mathrm{PM} \\
\text { Dry matter feed intake, } \mathrm{kg} D M / \mathrm{kg} M W\end{array}$} & $\begin{array}{c}\text { Primípara } \\
\text { Heifer }\end{array}$ & $0,157^{\mathrm{a}}$ & $0,152^{\mathrm{ab}}$ & $0,146^{\mathrm{b}}$ \\
\hline & $\begin{array}{l}\text { Multípara } \\
\text { Cow }\end{array}$ & $0,143^{\mathrm{a}}$ & $0,131^{b}$ & $0,144^{\mathrm{a}}$ \\
\hline \multirow{2}{*}{$\begin{array}{l}\text { Produção de leite, } \mathrm{kg} \text { leite/dia } \\
\text { Milk yield, } \mathrm{kg} \text { milk/day }\end{array}$} & $\begin{array}{l}\text { Primípara } \\
\text { Heifer }\end{array}$ & $21,3^{\mathrm{a}}$ & $18,7^{b}$ & $19,6^{\mathrm{b}}$ \\
\hline & $\begin{array}{l}\text { Multípara } \\
\text { Cow }\end{array}$ & $20,0^{\mathrm{b}}$ & $20,3^{b}$ & $21,7^{\mathrm{a}}$ \\
\hline
\end{tabular}

a,b Médias seguidas de letras distintas na mesma linha diferem $(p<0,01)$ pelo teste de Bonferroni.

$a, b$ Averages followed by distinct letters in the same row differ $(p<.01)$ by Bonferroni test.

$\mathrm{MS}=$ matéria seca, $\mathrm{PM}=$ peso metabólico.

$D M=$ dry matter, $M W=$ metabolic weight .

climas quentes é a diminuição do consumo de matéria seca (Maust et al., 1972; McDowell et al., 1976; Chen et al., 1993). Neste trabalho, no entanto, houve aumento de $8,5 \%$ na PL das multíparas do tratamento IT em relação ao ICO, sem que houvesse diferença significativa no consumo de MS entre estes dois tratamentos. A análise do ambiente mostrou que estas duas instalações também não apresentaram diferenças nas médias do ITGU. Esses resultados indicaram que possivelmente ocorreu um benefício do sombreamento adicional proporcionado pela tela, traduzido na maior produção de leite. Porém, tal constatação merece investigações adicionais, principalmente no que se refere ao comportamento desses animais, pois outro tipo de estresse que não os ligados à termodinâmica do ambiente podem ter interferido nestes resultados. Neste ponto, ressalta-se a importância do tamanho da área de sombreamento disponível às vacas leiteiras, o que, provavelmente, possibilitou maior conforto térmico.

Outros autores, como Collier et al. (1981) e Roman-Ponce et al. (1977), relataram aumentos de 17 e 10,7\%, respectivamente, na produção de leite diária com o sombreamento dos animais. Todavia, é importante ressaltar que estes trabalhos compararam vacas com ausência e disponibilidade total de sombra, o que não foi o caso do presente estudo.

Os resultados de PL das multíparas dos trata- mentos ICO e ICL estão de acordo com os relatos de Furquay (1997), que, em condições climáticas semelhantes às do presente estudo (Tbs de 24 a $33^{\circ} \mathrm{C}$ e UR de 70 a 90\%), também não observou diferenças significativas na produção de leite, em vacas submetidas a tratamentos com e sem climatização. Entretanto, os mesmos autores concluíram que as vacas do grupo controle apresentaram menor persistência da lactação. Isso é indicativo de que a persistência de lactação é um parâmetro importante no que se refere à investigação da eficiência da climatização em vacas lactantes, uma vez que persistências mais curtas afetam negativamente a produção de leite por vaca ano.

O menor consumo de MS pelas vacas do tratamento ICL em relação ao ICO, não foi traduzido em menor produção, uma vez que a PL em ICO e ICL foram iguais. De forma semelhante, Damasceno\& Targa (1998) compararam a produção de leite e o consumo alimentar de animais alojados em diferentes instalações e não encontraram diferenças no consumo de MS, embora tenham relatado diferença de $8,1 \%$ na produção de leite nos diferentes tratamentos.

No que se refere à análise física do ambiente, a média diária do ITGU foi significativamente menor $(p<0,01)$ no ICL em relação ao ICO e IT, porém isso não refletiu em maior consumo ou produção de leite naquela instalação. 


\section{Conclusões}

As primíparas apresentaram freqüências respiratórias e temperaturas retais mais altas que as multíparas nos horários mais quentes do dia, o que indicou condição de estresse mínimo para aquela categoria. Na instalação climatizada, no horário mais quente do dia, as primíparas apresentaram temperatura de pele mais alta, o que sugere a dominância das multíparas com relação à disputa por espaço no local climatizado.Tais resultados sugerem estudos adicionais sobre a fisiologia e o comportamento de multíparas e primíparas submetidas ao mesmo ambiente.

A freqüência respiratória de todos os animais da instalação climatizada foi significativamente menor, se comparada à das outras duas instalações, em todos os horários.

A utilização da tela como sombreamento apresentou resultado melhor, se comparado aos outros dois tratamentos, em termos de produção de leite para as multíparas.

O índice de temperatura e umidade entre 75 e 76, apesar de considerado estressante por diversas fontes da literatura, não foi associado à condição de estresse pelos animais. Tal fato sugere que maior número de investigações acerca dos valores críticos desses índices deve ser feito em condições de clima tropical, para o estabelecimento de parâmetros mais adequados, o que permitiria a escolha do índice que refletisse com maior precisão o estresse térmico nos animais.

\section{Agradecimento}

À Fundação de Amparo à Pesquisa do Estado de São Paulo (FAPESP) e à Coordenadoria de Apoio à Pesquisa e Ensino Superior (CAPES), pelo apoio financeiro, bem como à Prefeitura do Campus da USP em Pirassununga, pela cessão dos animais e das instalações.

\section{Literatura Citada}

AGUIAR, I.S. Respostas termorreguladoras, armazenamento de calor corporal e produção de leite de vacas holandesas mantidas ao sol e com acesso à sombra natural. Botucatu: Universidade Estadual Paulista, 1999. 76p. Tese (Doutorado) - Universidade Estadual Paulista, 1999.

ALBRIGHT, J.L. Feeding behaviour of dairy cattle. Journal of Dairy Science, v.76, p.485-491, 1993.

ANDERSSON, B.E.; JÓNASSON, H. Regulação da temperatura e fisiologia ambiental. In: SWENSON, M.J. (Ed.) Dukes - fisiologia dos animais domésticos. 11.ed. Rio de Janeiro: Guanabara Koogan, 1996. p.805-841.

ARCARO JR., I.; ARCARO, J.R.P.; POZZI, C.R. et al. Produção e composição do leite de vacas holandesas em sala de espera climatizada. In: CONGRESSO BRASILEIRO DE BIOMETEOROLOGIA, 3., 2001, Maringá. Anais... Maringá: Sociedade Brasileira de Biometeorologia, 2001. p.368.

BACCARI JR., F.; RAMOS, A.A.; VILLARES, JB. Temperaturas internas e externas de bovinos chianina e zebuínos Nelore. In: CONGRESSO INTERNACIONAL DA RAÇA CHIANINA, 2., 1978, São Paulo. Anais... São Paulo, 1978. p. 147-153.

BACCARI JR., F.; CAMPOS NETO, O.; ROCHA, G.P. Variação fisiológica da temperatura retal das 8 às 18 horas em bovinos holandeses - correlação com a temperatura ambiente e hora do dia. In: JORNADA CIENTÍFICA DA ASSOCIAÇÃO DOS DOCENTES DO CÂMPUS DE BOTUCATU, 7., 1979, Botucatu. Anais... Botucatu: Universidade Estadual Paulista, 1979. p.5-8.

BACCARI JR., F.; ASSIS, P.S.; POLASTRE, R. et al. Shade management in tropical environment for milk production in crossbred cows. Proceedings Western Section American Society of Agricultural Engineering, v.33, p.209-210, 1982.

BACCARI JR., F.; FRÉ, C.A.; ASSIS, R.S. et al. Valores fisiológicos da temperatura retal em vacas holandesas em clima tropical de altitude. In: ENCONTRO DE PESQUISAS VETERINÁRIAS, 1., 1984, Londrina. Anais... Londrina: 1984. p.15-22.

BACCARI JR., F. Manejo ambiental para produção de leite em climas quentes. In: CONGRESSO BRASILEIRO DE BIOMETEOROLOGIA, 3., 1998, Goiânia. Anais... Goiânia: Sociedade Brasileira de Biometeorologia, 1998. p.136-161.

BACCARI JR., F. Manejo ambiental da vaca leiteira em climas quentes. Londrina: Universidade Estadual de Londrina, 2001. 142p.

BUFFINGTON, D.E.; COLLAZO-AROCHO, A.; CANTON, G.H. et al. Black globe-humidity index (ITGU) as comfort equation for dairy cows. St. Joseph, MI, USA. Transactions of American Society of Agricultural Engineering, v.24, n.3, p.711-14, 1981.

CAPPA, V.; VAZHAPILLY, P.; MAIANTI, M.G. et al. Effect of environmental variations (microclimate) on the performance of dairy cows. Scienza e Tecnica LatieroCasearia, v.40, p.98-115, 1989.

CARDOSO, R.M.; FALCO, J.E.; SILVA, A.M. et al. Reações fisiológicas de vacas leiteiras mantidas à sombra, ao sol e em ambiente parcialmente sombreado. Revista Sociedade Brasileira de Zootecnia, v.12, n.3, p.458-468, 1983.

CHEN, K.H.; HUBER, J.T.; SIMAS, J. et al. Effect of protein quality and evaporative cooling on lactational performance of Holstein cows in hot weather. Journal of Dairy Science, v.76, n.3, p.816-825, 1993.

COLLIER, R.J.; ELEY, R.M.; SHARMA, A.K. et al. Shade management in subtropical environment for milk yield and composition in Holstein an Jersey cows. Journal of Dairy Science, v.64, p.844-849, 1981.

COLLIER, R.J.; BEEDE, D.K. Thermal stress as a factor associated with nutrient requirements and interrelationships. In: McDOWELL, L.R. (Ed.) Animal feeding and nutrition - a series of monographs. Orlando: Academic Press, 1985. p.59-71.

DAMASCENO, J.C.; TARGA, L.A. Definição de variáveis climáticas na determinação da resposta de vacas holandesas

R. Bras. Zootec., v.33, n.1, p.181-191, 2004 
em um sistema “free-stall”, Engenharia na Agricultura, v.12, n.2, p.12-25, 1998.

FEHR, R.L.; PRIDDY, K.T.; McNEILL, S.G. et al. Limiting swine stress with evaporative cooling in the southeast. Transactions of American Society of Agricultural Engineering, v.26, n.4, p.542-545, 1993.

FURQUAY, J.W. Heat stress as if affects animal production. Livestock Environment, v.2, p.1133-1137, 1997.

HAHN, G.L.; PARKHURRST, A.M.; GAUGHAN, J.B. Cattle respiration rate as a function of ambient temperature. Transactions of American Society of Agricultural Engineering, v.40, p.97-121, 1997.

JOHNSON, H.D. Environmental management of cattle to minimize the stress of climatic change. International Journal of Biometeorology, v.24, p.65-78, 1980.

JOHNSON, H.D. Role of physiology in cattle production in the tropics. New York: Praeger Scientific, 1982. 212p.

KOLB, E. Fisiologia veterinária. 4.ed. Rio de Janeiro: Guanabara Koogan, 1987. 612p.

LEMERLE, C.; GODDARD, M.E. Assessment of heat stress in dairy cattle in Papua New Guinea. Animal Health Production, v.18, p.232-242, 1986.

MAUST, L.E.; McDOWELL, R.E.; HOOVEN, N.W. Effect of summer weather on performance of Holstein cows in three stages of lactation. Journal of Dairy Science, v.55, p.1133-1139, 1972.

McDOWELL, R.E.; HOOVEN, N.M.; CAMOENS, J.K. Effect of climate on performance of Holstein in first lactation. Journal of Dairy Science, v.59, n.5, p.965-973, 1976.

McGUIRE, M.A.; BEEDE, D.K.; DELORENZO, M.A. Effects of thermal stress and level of feed intake on portal plasma flow and net fluxes of metabolites in lactating Holstein cows. Journal of Animal Science, v.67, p.1050-1060, 1989.
MOTA, L.S.L.S. Adaptação e interação genótipo-ambiente em vacas leiteiras. Ribeirão Preto: Universidade de São Paulo, 1997. Tese (Doutorado em Ciências) - Universidade de São Paulo, 1997.

NÄÄS, I.A. Princípios de conforto térmico na produção animal. São Paulo: Ícone, 1989. 183p.

NÄAS, I.A.; ARCARO JR., I. Influência de ventilação e aspersão em sistemas de sombreamento artificial para vacas em lactação em condições de calor. Revista Brasileira de Engenharia Agrícola e Ambiental, v.5, n.1, p.139-142, 2001.

ROMAN-PONCE, H.; THATCHER, W.W.; BUFFINGTON, D.E. et al. Physiological and production responses of dairy cattle to a shade structure in a subtropical environment. Journal of Dairy Science, v.60, p.424-30, 1977.

STATISTICAL ANALYSES SYSTEM - SAS. SAS/STAT user's guide. Version 6, 4.ed., v.2, Cary: 1990.

SCHNEIDER, P.L.; BEEDE, D.K.; WILCOX, C.J. et al. Influence of dietary sodium and potassium bicarbonate and total potassium on heat stressed lactating cows. Journal of Dairy Science, v.67, p.2548-2553, 1984.

SILVA, R.G. Introdução à bioclimatologia animal. São Paulo: Nobel, 2000. 286p.

TITTO, E.A.L. Clima: Influência na produção de leite. In: SIMPÓSIO BRASILEIRO DE AMBIÊNCIA NA PRODUÇÃO DE LEITE, 1., 1998, Piracicaba. Anais... Piracicaba: Fundação de Estudos Agrários Luiz de Queiroz, 1998. p.10-23.

Recebido em: 03/10/02

Aceito em: 07/07/03 